\title{
Surgery for Esophageal Cancer: Quality of Life Matters
}

\author{
Jonathan C Salo, MD, FACS \\ Department of Surgery, Carolinas Medical Center, Charlotte, NC
}

Esophageal cancer and its treatment have a profound impact not only on a patient's length of life, but quality of life as well. Dysphagia, weight loss, and eating restrictions are frequent sequelae of both the disease and its treatment.

In this issue, investigators from the Academic Medical Center in Amsterdam examined quality of life before and after resection for distal esophageal cancers. ${ }^{1}$ They found that among preoperative patients, the quality-of-life physical symptom scale predicted postoperative overall survival independent of the tumor length and endoscopic ultrasound T-stage. After surgical resection, the quality-of-life pain scale, social function, and activity level predicted overall survival independent of pathological T-stage and N-stage.

Clearly, self-reported health-related quality of life (HRQoL) adds predictive value to our best available preoperative and postoperative prognosticators. The challenge is how to use this information.

One important benefit of quality-of-life research is to help evaluate the outcomes of different therapies, such as the analysis by the same investigators who examined quality of life after transhiatal compared with transthoracic esophagectomy. ${ }^{2}$

It is intriguing to speculate about what mechanisms may underlie the effects of HRQoL on overall and diseasespecific survival. These speculations may help to generate hypotheses for further investigation that may help further understand tumor biology.

One mechanism of these interactions may be that quality of life is a marker for occult metastatic disease. Recent advances in serum proteonomics in gastrointestinal cancer patients suggest that even among patients thought to have localized disease, cancer-associated proteins can be

(C) Society of Surgical Oncology 2009

First Received: 7 September 2009;

Published Online: 23 October 2009

J. C. Salo, MD, FACS

e-mail: jonathan.salo@carolinashealthcare.org detected within the serum. ${ }^{3}$ It would be reasonable to assume that even localized tumors, in patients with imaging negative for distant disease, might elaborate serum factors that would adversely affect quality of life. As this field grows and specific proteins are identified, it will be interesting to see whether the age-old problem of cancerassociated cachexia can be more fully understood.

Health-related quality of life may also be a window into the patient's host response to their neoplasm. Social function, activity level, and pain may be surrogates for their ability to recover from surgery and the presence of major or subclinical surgical complications. Postoperative decrements in quality of life may also be a marker for postoperative complications, which are known to affect quality of life and alter prognosis, even subsequent to the perioperative period. ${ }^{4,5}$

On the other hand, social function and activity level may be related to cancer-related alterations in circadian rhythms. Circadian rhythm alterations and social function are related in complex ways: social cues help to reset the biological clock, while disruptions in circadian and sleep cycle patterns tend to affect social interactions. It been shown, for instance, that cancer patients exhibit abnormalities in circadian rhythm function. ${ }^{6}$ Disruption in circadian rhythm function in animals (by ablation of the suprachiasmatic nucleus) leads to faster tumor growth, presumably through neuroimmunological pathways. ${ }^{7}$ In a recent study of 192 patients with metastatic colon cancer, circadian rhythm dysfunction was measured with a 24-h actigraph affixed to a patient's wrist. Alterations in circadian rhythm were associated with shorter survival and worse measures of global quality of life, social function, fatigue, and pain. ${ }^{8}$

The poor prognosis associated with postoperative pain may also be an indicator of the complex relationship between pain and cancer biology. The presence of pain appears to induce immune dysfunction, which may affect tumor growth. An intriguing report involves the initial 
intravenous injection of tumor cells into mice, with resulting liver metastases. After tumor injection, laparotomy was performed with inhalation general anesthesia either alone or supplemented with spinal blockade. ${ }^{9}$ In those mice treated with spinal blockade, fewer liver metastases developed. Immunological studies documented better preservation of natural killer cell activity and Th1/ Th2 balance in animals treated with spinal blockade. While these possible mechanisms have not been demonstrated in clinical research, they do help to generate testable hypotheses in human cancer research.

In summary, the article in this issue of Annals of Surgical Oncology emphasizes the growing awareness that health-related quality of life may not only play a role in providing prognostic information for cancer patients, but may give us some insight into cancer biology.

\section{REFERENCES}

1. van Heijl M, Sprangers M, de Boer A, et al. Preoperative and early postoperative quality of life predict survival in potentially curable patients with esophageal cancer. Ann Surg Oncol. doi: 10.1245/s10434-009-0731-y.

2. de Boer AG, van Lanschot JJ, van Sandick JW, Hulscher JB, Stalmeier PF, de Haes JC, et al. Quality of life after transhiatal compared with extended transthoracic resection for adenocarcinoma of the esophagus. J Clin Oncol. 2004;22:4202-8.

3. Poon TC, Sung JJ, Chow SM, Ng EK, Yu AC, Chu ES, et al. Diagnosis of gastric cancer by serum proteomic fingerprinting. Gastroenterology. 2006;130:1858-64.

4. Rutegard M, Lagergren J, Rouvelas I, Lindblad M, Blazeby JM, Lagergren P. Population-based study of surgical factors in relation to health-related quality of life after oesophageal cancer resection. Br J Surg. 2008;95:592-601.

5. Ott K, Bader FG, Lordick F, Feith M, Bartels H, Siewert JR. Surgical factors influence the outcome after Ivor-Lewis esophagectomy with intrathoracic anastomosis for adenocarcinoma of the esophagogastric junction: a consecutive series of 240 patients at an experienced center. Ann Surg Oncol. 2009;16:1017-25.

6. Sephton S, Spiegel D. Circadian disruption in cancer: a neuroendocrine-immune pathway from stress to disease? Brain Behav Immun. 2003;17:321-8.

7. Filipski E, King VM, Li X, Granda TG, Mormont MC, Liu X, et al. Host circadian clock as a control point in tumor progression. J Natl Cancer Inst. 2002;94:690-7.

8. Innominato PF, Focan C, Gorlia $\mathrm{T}$, Moreau $\mathrm{T}$, Garufi $\mathrm{C}$, Waterhouse $\mathrm{J}$, et al. Circadian rhythm in rest and activity: a biological correlate of quality of life and a predictor of survival in patients with metastatic colorectal cancer. Cancer Res. 2009; 69:4700-7.

9. Wada H, Seki S, Takahashi T, Kawarabayashi N, Higuchi H, Habu Y, et al. Combined spinal and general anesthesia attenuates liver metastasis by preserving TH1/TH2 cytokine balance. Anesthesiology. 2007; 106:499-506. 